\title{
ChemComm
}

Check for updates

Cite this: Chem. Commun., 2018 54, 3544

DOI: $10.1039 / \mathrm{c} 8 \mathrm{cc} 90117 a$

rsc.li/chemcomm

\section{Correction: Jadomycins, put a bigger ring in it: isolation of seven- to ten-membered ring analogues}

\author{
Stephanie M. Forget, ${ }^{a}$ Camilo F. Martinez-Farina ${ }^{a}$ and David L. Jakeman*b
}

Correction for 'Jadomycins, put a bigger ring in it: isolation of seven- to ten-membered ring analogues' by Camilo F. Martinez-Farina et al., Chem. Commun., 2015, 51, 14617-14619.

The authors regret that an incorrect structure was reported in the original article. Herein, the authors report a revised structure for $\mathbf{3}$, the jadomycin incorporating 7-aminoheptanoic acid. The revised structure deviates from the other jadomycin congeners, $\mathbf{1}$ derived from aminobutyric acid, and 2 from aminohexanoic acid, reported in the series by the absence of a cyclized E-ring, and by the attachment of a C-linked $\mathrm{CH}_{2}-\mathrm{CH}_{2}-\mathrm{OH}$ moiety at the 3a position. Re-assignment of the molecule was supported by NMR spectroscopic data and HRMS data. A ten-membered ring containing jadomycin (jadomycin Dec) was not fully characterized.

Stephanie M. Forget has been added as an author as she was involved in data analysis resulting in the structural revision, updating the relevant characterization data and writing the text for the correction.

On pages 14618-14619, the paragraph beginning "While characterizing the isolated jadomycin analogues..." should be replaced by the following text:

"The following discussion uses the chemical shifts from the major compound. By recording the ${ }^{1} \mathrm{H}$ NMR spectrum using a more dilute sample of 3 , the issue of broadness that was observed in the previously reported proton spectrum was ameliorated, allowing key coupling constants to be observed. The 3a proton $(4.72 \mathrm{ppm})$ was identified as a doublet of doublets, suggesting a vicinal proton coupling partner; analysis of the COSY NMR spectrum revealed that there was a strong cross-peak between 3a- $\mathrm{H}$ and diastereotopic methylene protons ( $\mathrm{H}-2,1.92$ and $1.77 \mathrm{ppm})$, which were in turn coupled to a second methylene group ( $\mathrm{H}-1,3.48$ and $3.40 \mathrm{ppm})$. These data provided evidence for a $-\mathrm{CH}-\mathrm{CH}_{2}-\mathrm{CH}_{2}-\mathrm{OH}$ spin system. Of significance was the observed chemical shift for the $3 \mathrm{a}$ carbon at $64.3 \mathrm{ppm}$, which was different from the carbon shifts reported for $1(92.5 \mathrm{ppm})$ and $2(92.6 \mathrm{ppm})$, but was close to the shifts reported for other C-branched jadomycins. ${ }^{1}$ Complete NMR assignments for 3 can be found in the ESI. The minor compound present in the sample was not fully characterized due to substantial signal overlap with the major compound and due to low signal intensities in the $2 \mathrm{D}$ spectra. Therefore, we do not propose a structure for the secondary compound. The 1D-ROESY data for the 3a proton provided evidence of through-space correlation to the H-1 and H-2 protons, consistent with the revised structure (Fig. 4). In addition to these data, a mass spectrum in negative mode was collected. The major signal from the ESI negative MS spectrum ([M $-\mathrm{H}]^{-}$ $608 \mathrm{~m} / \mathrm{z}$ ) was consistent with the proposed structure herein. HRMS data, $[\mathrm{M}-\mathrm{H}]^{-} \mathrm{C}_{33} \mathrm{H}_{38} \mathrm{NO}_{10}$ found 608.2505, calculated 608.2496, was consistent with 3 . To address the fact that the HRMS for the initially proposed structure (ten-membered ring) was observed, we propose that the sample submitted for HRMS analysis likely contained trace amounts of other species, as was evident when expanding the baseline in the proton NMR spectrum. It is likely that the ten-membered ring initially proposed (jadomycin Dec) was present in the analyzed sample, but was not the major component. A qualitative observation of the distinctive colour of 3 in solution, a violet purple in methanol, was consistent with the colour reported for the other C-branched jadomycins. ${ }^{1}$ Consistently, the $\lambda_{\max }$ for $3(551 \mathrm{~nm})$ was distinct from those reported for $1(524 \mathrm{~nm})$ and $2(522 \mathrm{~nm})$. ."

On page 14619, the paragraph beginning "The production and isolation of compounds 1-3..." should be replaced by the following text:

"The isolation of compounds 1 and $\mathbf{2}$ demonstrates the first examples of jadomycin analogues incorporating seven- and ninemembered rings. Re-evaluation of the fractions obtained from the culture from which $\mathbf{3}$ was isolated did not result in the identification of a ten-membered ring containing jadomycin (jadomycin Dec), which was, therefore, not produced in sufficient quantity for isolation and subsequent characterization."

\footnotetext{
${ }^{a}$ Department of Chemistry, Dalhousie University, Halifax, Nova Scotia B3H 4R2, Canada

${ }^{b}$ College of Pharmacy, Dalhousie University, Halifax, Nova Scotia B3H 4R2, Canada. E-mail: david.jakeman@dal.ca
} 
On page 14617, structure 3 in Scheme 1 was incorrect. The correct structure is shown in Fig. 1.

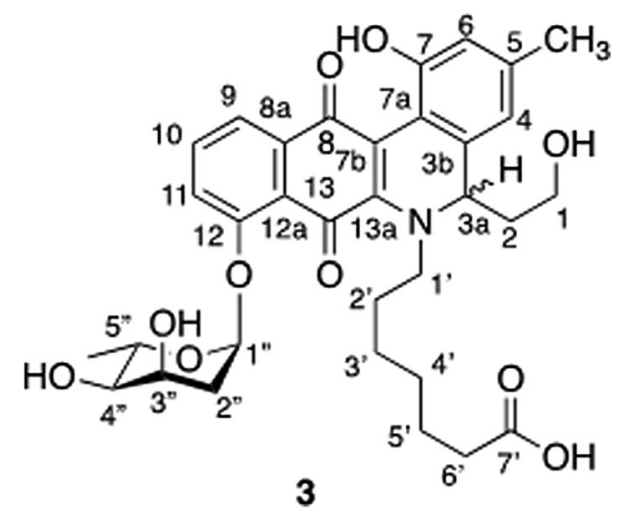

Fig. 1 Corrected structure for compound 3.

On page 14619, the bottom spectrum in Fig. 4 was incorrectly labelled. The correctly labelled spectrum is shown in Fig. 2.

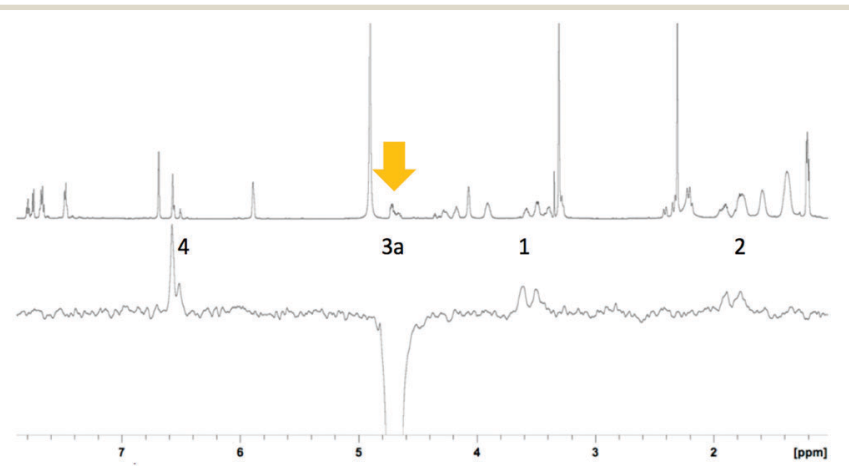

Fig. 2 Corrected labelling of the bottom spectrum in Fig. 4 of the original article.

The Royal Society of Chemistry apologises for these errors and any consequent inconvenience to authors and readers.

\section{References}

1 A. W. Robertson, J. M. MacLeod, L. W. MacIntyre, S. M. Forget, S. R. Hall, L. G. Bennet, H. Correa, R. G. Kerr, K. B. Goralski and D. L. Jakeman, J. Org. Chem., 2018, DOI: 10.1021/acs.joc.7b02823. 